\title{
NEW CHARACTERIZATION OF 2-PRE-HILBERT SPACE
}

\author{
Samoil Malčeski ${ }^{1}$, Katerina Anevska ${ }^{2}$ and Risto Malčeski ${ }^{3}$
}

\begin{abstract}
The problem of finding necessary and sufficient conditions a 2-normed space to be treated as 2-pre-Hilbert space is the focus of interest of many mathematicians. Few characterizations of 2-inner product are given in [1], [3], [5], [6], [8] and [9]. In this paper a new necessary and sufficient condition for existence of 2-inner product into 2normed space is given.
\end{abstract}

\section{INTRODUCTION}

Let $L$ be a real vector space with dimension greater than 1 and $\|\cdot, \cdot\|$ be a real function on $L \times L$ such that:

a) $\|x, y\| \geq 0$, for all $x, y \in L$ and $\|x, y\|=0$ if and only if the set $\{x, y\}$ is linearly dependent,

b) $\|x, y\|=\|y, x\|$, for all $x, y \in L$,

c) $\|\alpha x, y\|=|\alpha| \cdot\|x, y\|$, for all $x, y \in L$ and for each $\alpha \in \mathbf{R}$, and

d) $\|x+y, z\| \leq\|x, z\|+\|y, z\|$, for all $x, y, z \in L$.

The function $\|\cdot, \cdot\|$ is said to be 2-norm of $L$, and $(L,\|\cdot, \cdot\|)$ is said to be vector 2-normed space ([7]). The inequality in the axiom $d$ ) is said to be parallelepiped inequality.

Let $n>1$ be a positive integer, $L$ be a real vector space, $\operatorname{dim} L \geq n$ and $(\cdot, \cdot \mid \cdot)$ be a real function over $L \times L \times L$ such that:

i) $(x, x \mid y) \geq 0$, for all $x, y \in L$ and $(x, x \mid y)=0$ if and only if $x$ and $y$ are linearly dependent,

ii) $(x, y \mid z)=(y, x \mid z)$, for all $x, y, z \in L$,

iii) $(x, x \mid y)=(y, y \mid x)$, for all $x, y \in L$,

iv) $(\alpha x, y \mid z)=\alpha(x, y \mid z)$, for all $x, y, z \in L$. and for each $\alpha \in \mathbf{R}$, and

v) $\left(x+x_{1}, y \mid z\right)=(x, y \mid z)+\left(x_{1}, y \mid z\right)$, for all $x_{1}, x, y, z \in L$.

The function $(\cdot, \cdot \mid \cdot)$ is said to be 2-inner product, and $(L,(\cdot, \cdot \mid \cdot))$ is said to be 2-preHilbert space ([3]). 
The concepts of 2-norm and 2-inner product are two dimensional analogies of the concepts of norm and inner product. R. Ehret proved ([7]) that if $(L,(\cdot, \cdot \mid \cdot))$ is a 2-preHilbert space, then

$$
\|x, y\|=(x, x \mid y)^{1 / 2},
$$

for all $x, y \in L$ defines 2-norm. So, we get vector 2-normed space $(L,\|\cdot, \cdot\|)$ and moreover, for all $x, y, z \in L$ the following equalities are satisfied:

$$
\begin{aligned}
& (x, y \mid z)=\frac{\|x+y, z\|^{2}-\|x-y, z\|^{2}}{4}, \\
& \|x+y, z\|^{2}+\|x-y, z\|^{2}=2\left(\|x, z\|^{2}+\|y, z\|^{2}\right),
\end{aligned}
$$

The equality (3) is actually analogous to the parallelogram equality and it is called parallelepiped equality. Further, 2-normed space $L$ is 2-pre-Hilbert space if and only if for all $x, y, z \in L$ the equality (3) holds true.

\section{Characterization OF 2-PRE-HiLbert SPACE}

The problem of characterization of 2-pre-Hilbert spaces, i.e. finding the necessary and sufficient conditions the 2-normed spaces to be treated as 2-pre-Hilbert space is of particular interest while studying the 2-normed spaces. Thus, in [1] is given characterization of 2-pre-Hilbert space using the equality of Euler-Lagrange type, in [8] is given characterization using the strictly convex norm with modulus $c$, and in [9] are given characterizations using the Mercer inequality for 2-normed space and its equivalent inequality. In the following theorem are given some of the already known characterizations of 2-pre-Hilbert spaces, which are necessary for our further considerations.

Theorem 1 ([3]). Let $(L,\|\cdot, \cdot\|)$ be 2-normed space. $L$ is 2-pre-Hilbert space if and only if for each $z \in L \backslash\{0\}$ one of the following conditions is satisfied:

$I I_{1}$. For all $x, y, z \in L$ such that $\|x, z\|=\|y, z\|$ and for all $m, n \in \mathbf{R}$ it holds true that

$$
\|m x+n y, z\|=\|n x+m y, z\| .
$$

$I I_{2} .\|x+y, z\|=\|x-y, z\|, x, y, z \in L$ implies that

$$
\|x+y, z\|^{2}=\|x, z\|^{2}+\|y, z\|^{2}
$$

$I I_{3}$. It exists a real number $\alpha \neq 0, \pm 1$ such that $\|x, z\|=\|y, z\|, x, y, z \in L$ implies that $\|x+\alpha y, z\|=\|\alpha x+y, z\|$.

$I I_{4}$. It exists a real number $\alpha \neq 0, \pm 1$ such that $\|x+y, z\|=\|x-y, z\|, \quad x, y, z \in L$ implies that $\|x+\alpha y, z\|=\|x-\alpha y, z\|$.

$I_{5}$. $\|x, z\|=\|y, z\|, x, y, z \in L$ implies that for each real number $\alpha>0$ it holds true that 


$$
\left\|\alpha x+\alpha^{-1} y, z\right\| \geq\|x+y, z\| .
$$

$I I_{6}$. For all $x_{1}, x_{2}, x_{3}, z \in L$ such that $\sum_{i=1}^{3} x_{i}=0$ and $\left\|x_{1}, z\right\|=\left\|x_{2}, z\right\|$ it holds true that

$$
\left\|x_{1}-x_{3}, z\right\|=\left\|x_{2}-x_{3}, z\right\| \text {. }
$$

$I I_{7}$. For all $x_{1}, x_{2}, x_{3}, x_{4}, z \in L$ such that $\sum_{i=1}^{4} x_{i}=0$ and $\left\|x_{1}, z\right\|=\left\|x_{2}, z\right\|$ and $\left\|x_{3}, z\right\|=$ $\left\|x_{4}, z\right\|$ it holds true that

$$
\left\|x_{1}-x_{3}, z\right\|=\left\|x_{2}-x_{4}, z\right\| \text { and }\left\|x_{2}-x_{3}, z\right\|=\left\|x_{1}-x_{4}, z\right\| .
$$

$I I_{8}$. For all $x_{1}, x_{2}, x_{3}, z \in L$ the value of the expression

$$
\begin{aligned}
F\left(x_{1}, x_{2}, x_{3}, z\right)= & \left\|x_{1}+x_{2}+x_{3}, z\right\|^{2}+\left\|x_{1}+x_{2}-x_{3}, z\right\|^{2}- \\
& -\left\|x_{1}-x_{2}-x_{3}, z\right\|^{2}-\left\|x_{1}-x_{2}+x_{3}, z\right\|^{2}
\end{aligned}
$$

does not depend on $x_{3}$.

$I I_{9}$. For all $x_{1}, \ldots, x_{n}, z \in L, n \geq 3$ such that $\sum_{i=1}^{n} x_{i}=0$ it holds true that

$$
\sum_{i \neq k}\left\|x_{i}-x_{k}, z\right\|^{2}=2 n \sum_{i=1}^{n}\left\|x_{i}, z\right\|^{2} \text {. }
$$

In the following theorem a new characterization of 2-pre-Hilbert space will be given.

Theorem 2. Let $(L,\|\cdot, \cdot\|)$ be a real 2-normed space. Then $L$ is 2-pre-Hilbert space if and only if the following condition is satisfied

$I I_{10}$. If $n \geq 3, x_{1}, x_{2}, \ldots, x_{n}, z \in L$ and $\alpha_{1}, \alpha_{2}, \ldots, \alpha_{n}$ are real numbers such that $\sum_{i=1}^{n} \alpha_{i}=0$, then

$$
\left\|\sum_{i=1}^{n} \alpha_{i} x_{i}, z\right\|^{2}=-\sum_{1 \leq i<j \leq n} \alpha_{i} \alpha_{j}\left\|x_{i}-x_{j}, z\right\|^{2} .
$$

Proof. Let the condition $I I_{10}$ be satisfied. If $x_{1}, x_{2}, z \in L$, then for $x_{3}=0$ and the condition $I I_{10}$ applied to the vectors $x_{1}, x_{2}, x_{3}, z \in L$ and the real numbers $\alpha_{1}=\alpha_{2}=1, \alpha_{3}=-2$ follow the following equalities

$$
\begin{aligned}
\left\|x_{1}+x_{2}, z\right\|^{2} & =\left\|x_{1}+x_{2}+(-2) \cdot 0, z\right\|^{2} \\
& =-1 \cdot(-2)\left\|x_{1}-0, z\right\|^{2}-1 \cdot(-2)\left\|x_{2}-0, z\right\|^{2}-1 \cdot 1\left\|x_{1}-x_{2}, z\right\|^{2} \\
& =2\left\|x_{1}, z\right\|^{2}+2\left\|x_{2}, z\right\|^{2}-\left\|x_{1}-x_{2}, z\right\|^{2},
\end{aligned}
$$

The latter implies the parallelepiped equality, which actually means that $L$ is 2-preHilbert space. 
Let $L$ be 2-pre-Hilbert space. Applying the principle of mathematical induction we will prove that the condition $I I_{10}$ is satisfied. Let $n=3, \alpha_{1}, \alpha_{2}, \alpha_{3}$ be real numbers such that $\alpha_{1}+\alpha_{2}+\alpha_{3}=0$ and $x_{1}, x_{2}, x_{3}, z \in L$. Then by the properties of 2 -inner product and since $\alpha_{1}+\alpha_{2}=-\alpha_{3}$ we get that

$$
\begin{aligned}
&\left\|\alpha_{1} x_{1}+\alpha_{2} x_{2}+\alpha_{3} x_{3}, z\right\|^{2}=\left\|\alpha_{1}\left(x_{1}-x_{3}\right)+\alpha_{2}\left(x_{2}-x_{3}\right), z\right\|^{2} \\
&= \alpha_{1}^{2}\left\|x_{1}-x_{3}, z\right\|^{2}+\alpha_{1} \alpha_{2}\left(x_{1}-x_{3}, x_{2}-x_{3} \mid z\right) \\
&+\alpha_{1} \alpha_{2}\left(x_{2}-x_{3}, x_{1}-x_{3} \mid z\right)+\alpha_{2}^{2}\left\|x_{2}-x_{3}, z\right\|^{2} \\
&= \alpha_{1}^{2}\left\|x_{1}-x_{3}, z\right\|^{2}+\alpha_{1} \alpha_{2}\left(x_{1}-x_{3}, x_{1}-x_{3}+x_{2}-x_{1} \mid z\right) \\
&+\alpha_{1} \alpha_{2}\left(x_{2}-x_{3}, x_{2}-x_{3}+x_{1}-x_{2} \mid z\right)+\alpha_{2}^{2}\left\|x_{2}-x_{3}, z\right\|^{2} \\
&= \alpha_{1}^{2}\left\|x_{1}-x_{3}, z\right\|^{2}+\alpha_{1} \alpha_{2}\left\|x_{1}-x_{3}, z\right\|^{2}+\alpha_{1} \alpha_{2}\left(x_{1}-x_{3}, x_{2}-x_{1} \mid z\right) \\
&+\alpha_{1} \alpha_{2}\left\|x_{2}-x_{3}, z\right\|^{2}+\alpha_{1} \alpha_{2}\left(x_{2}-x_{3}, x_{1}-x_{2} \mid z\right)+\alpha_{2}^{2}\left\|x_{2}-x_{3}, z\right\|^{2} \\
&= \alpha_{1}\left(\alpha_{1}+\alpha_{2}\right)\left\|x_{1}-x_{3}, z\right\|^{2}+\alpha_{2}\left(\alpha_{1}+\alpha_{2}\right)\left\|x_{2}-x_{3}, z\right\|^{2} \\
&-\alpha_{1} \alpha_{2}\left[\left(x_{1}-x_{3}, x_{1}-x_{2} \mid z\right)+\left(x_{3}-x_{2}, x_{1}-x_{2} \mid z\right)\right] \\
&=-\alpha_{1} \alpha_{3}\left\|x_{1}-x_{3}, z\right\|^{2}-\alpha_{2} \alpha_{3}\left\|x_{2}-x_{3}, z\right\|^{2}-\alpha_{1} \alpha_{2}\left\|x_{1}-x_{2}, z\right\|^{2},
\end{aligned}
$$

which means that the condition $I I_{10}$ holds true.

Let in the 2-pre-Hilbert space $L$ the condition $I_{10}$ be satisfied for some positive integer $n \geq 3$. Let $x_{1}, x_{2}, \ldots, x_{n}, x_{n+1}, z \in L$ and $\alpha_{1}, \alpha_{2}, \ldots, \alpha_{n}, \alpha_{n+1}$ be real numbers such that $\sum_{i=1}^{n+1} \alpha_{i}=0$ and let

$$
\beta=\alpha_{1}+\alpha_{2}+\ldots+\alpha_{n-1}=-\left(\alpha_{n}+\alpha_{n+1}\right) .
$$

Then, since

$$
\frac{\beta}{-\alpha_{n+1}}+\frac{\alpha_{n}}{-\alpha_{n+1}}-1=0 \text { and } 1+\sum_{i=1}^{n+1} \frac{\alpha_{i}}{\beta}=0
$$

the inductive assumption implies that

$$
\begin{aligned}
& \left\|\sum_{i=1}^{n+1} \alpha_{i} x_{i}, z\right\|^{2}=\alpha_{n+1}^{2}\left\|\sum_{i=1}^{n-1} \frac{\alpha_{i}}{-\alpha_{n+1}} x_{i}+\frac{\alpha_{n}}{-\alpha_{n+1}} x_{n}+(-1) x_{n+1}, z\right\|^{2} \\
& \quad=\alpha_{n+1}^{2}\left\|\frac{\beta}{-\alpha_{n+1}}\left(\sum_{i=1}^{n-1} \frac{\alpha_{i}}{\beta} x_{i}\right)+\frac{\alpha_{n}}{-\alpha_{n+1}} x_{n}+(-1) x_{n+1}, z\right\|^{2} \\
& \quad=\alpha_{n+1}^{2}\left[-\frac{\beta \alpha_{n}}{\alpha_{n+1}^{2}}\left\|\sum_{i=1}^{n-1} \frac{\alpha_{i}}{\beta} x_{i}-x_{n}, z\right\|^{2}-\frac{\beta}{\alpha_{n+1}}\left\|\sum_{i=1}^{n-1} \frac{\alpha_{i}}{\beta} x_{i}-x_{n+1}, z\right\|^{2}-\frac{\alpha_{n}}{\alpha_{n+1}}\left\|x_{n+1}-x_{n}, z\right\|^{2}\right. \\
& \quad=-\beta \alpha_{n}\left\|\sum_{i=1}^{n-1} \frac{\alpha_{i}}{\beta} x_{i}-x_{n}, z\right\|^{2}-\beta \alpha_{n+1}\left\|\sum_{i=1}^{n-1} \frac{\alpha_{i}}{\beta} x_{i}-x_{n+1}, z\right\|^{2}-\alpha_{n} \alpha_{n+1}\left\|x_{n+1}-x_{n}, z\right\|^{2} \\
& \quad=-\beta \alpha_{n}\left[-\sum_{1 \leq i<j \leq n-1} \frac{\alpha_{i} \alpha_{j}}{\beta^{2}}\left\|x_{i}-x_{j}, z\right\|^{2}+\sum_{i=1}^{n} \frac{\alpha_{i}}{\beta}\left\|x_{i}-x_{n}, z\right\|^{2}\right]
\end{aligned}
$$




$$
\begin{aligned}
& -\beta \alpha_{n+1}\left[-\sum_{1 \leq i<j \leq n-1} \frac{\alpha_{i} \alpha_{j}}{\beta^{2}}\left\|x_{i}-x_{j}, z\right\|^{2}+\sum_{i=1}^{n} \frac{\alpha_{i}}{\beta}\left\|x_{i}-x_{n+1}, z\right\|^{2}\right] \\
& -\alpha_{n} \alpha_{n+1}\left\|x_{n+1}-x_{n}, z\right\|^{2} \\
= & \frac{\alpha_{n}+\alpha_{n+1}}{\beta} \sum_{1 \leq i<j \leq n-1} \alpha_{i} \alpha_{j}\left\|x_{i}-x_{j}, z\right\|^{2}-\sum_{i=1}^{n} \alpha_{i} \alpha_{n}\left\|x_{i}-x_{n}, z\right\|^{2} \\
& -\sum_{i=1}^{n} \alpha_{i} \alpha_{n+1}\left\|x_{i}-x_{n+1}, z\right\|^{2}-\alpha_{n} \alpha_{n+1}\left\|x_{n+1}-x_{n}, z\right\|^{2} \\
= & -\sum_{1 \leq i<j \leq n+1} \alpha_{i} \alpha_{j}\left\|x_{i}-x_{j}, z\right\|^{2} .
\end{aligned}
$$

The latter means that the condition $I I_{10}$ also holds true for $n+1$. So, the principle of mathematical induction implies that $I_{10}$ holds true for each positive integer.

The theorems 1 and 2 imply that in 2-normed space the conditions $I I_{1}-I I_{10}$ are equivalent to each other. In the further considerations we will prove that the condition $I I_{10}$ directly implies some of the conditions $I I_{1}-I I_{9}$.

Lemma 1. Let $L$ be 2-normed space. Then the condition $I I_{10}$ implies the condition $I I_{9}$.

Proof. Let $x_{1}, \ldots, x_{n}, z \in L, n \geq 3$ be such that $\sum_{i=1}^{n} x_{i}=0$. Then the condition $I I_{10}$ implies the following

$$
0=\left\|\sum_{i=1}^{n} x_{i}, z\right\|^{2}=\left\|x_{1}+x_{2}+\ldots+x_{n}-n \cdot 0, z\right\|^{2}=n \sum_{i=1}^{n}\left\|x_{i}-0, z\right\|^{2}-\sum_{1 \leq i<k \leq n}\left\|x_{i}-x_{k}, z\right\|^{2},
$$

which implies that

$$
\sum_{i \neq k}\left\|x_{i}-x_{k}, z\right\|^{2}=\sum_{1 \leq i<k \leq n}\left\|x_{i}-x_{k}, z\right\|^{2}+-\sum_{1 \leq k<i \leq n}\left\|x_{k}-x_{i}, z\right\|^{2}=2 n \sum_{i=1}^{n}\left\|x_{i}, z\right\|^{2},
$$

i.e. the condition $I_{9}$ is satisfied.

Lemma 2. Let $L$ be 2-normed space. Then the condition $I I_{10}$ implies the condition $I_{5}$. Proof. Let $\|x, z\|=\|y, z\|, x, y, z \in L$ and $\alpha>0$ be real number. Then the condition $I I_{10}$ implies the following

$$
\begin{aligned}
\left\|\alpha x+\alpha^{-1} y, z\right\|^{2} & =\left\|\alpha x+\left(-\alpha^{-1}\right)(-y)+\left(\alpha^{-1}-\alpha\right) 0, z\right\|^{2} \\
& =-\alpha\left(\alpha^{-1}-\alpha\right)\|x, z\|^{2}+\alpha^{-1}\left(\alpha^{-1}-\alpha\right)\|y, z\|^{2}+\|x+y, z\|^{2} \\
& =\left(-1+\alpha^{2}+\frac{1}{\alpha^{2}}-1\right)\|x, z\|^{2}+\|x+y, z\|^{2} \\
& =\left(\alpha+\frac{1}{\alpha}\right)^{2}\|x, z\|^{2}+\|x+y, z\|^{2} \geq\|x+y, z\|^{2},
\end{aligned}
$$


thus $\left\|\alpha x+\alpha^{-1} y, z\right\| \geq\|x+y, z\|$, i.e. the condition $I_{5}$ is satisfied.

Lemma 3. Let $L$ be 2-normed space. Then the condition $I I_{10}$ implies the condition $I I_{1}$. Proof. Let $x, y, z \in L$ be such that $\|x, z\|=\|y, z\| m, n \in \mathbf{R}$. Then the condition $I I_{10}$ implies that

$$
\begin{aligned}
\|m x+n y, z\|^{2} & =\|m x+n y+(-m-n) 0, z\|^{2} \\
& =m(m+n)\|x, z\|^{2}+n(m+n)\|y, z\|^{2}-m n\|x-y, z\|^{2}
\end{aligned}
$$

and

$$
\begin{aligned}
\|n x+m y, z\|^{2} & =\|n x+m y+(-m-n) 0, z\|^{2} \\
& =n(m+n)\|x, z\|^{2}+m(m+n)\|y, z\|^{2}-m n\|x-y, z\|^{2} .
\end{aligned}
$$

Further, since $\|x, z\|=\|y, z\|$, the last two equalities imply that

$$
\|n x+m y, z\|^{2}=\|m x+n y, z\|^{2} \text {, i.e. }\|m x+n y, z\|=\|n x+m y, z\|,
$$

The latter means that the condition $I_{1}$ is satisfied.

Lemma 4. Let $L$ be 2-normed space. Then the condition $I I_{10}$ implies the condition $I_{3}$.

Proof. Let $\|x, z\|=\|y, z\|, x, y, z \in L$ an let $\alpha$ be a real number such that $\alpha \neq 0, \pm 1$.

Then the condition $I I_{10}$ implies that

$$
\begin{aligned}
\|x-y, z\|^{2} & =\left\|\frac{1}{\alpha}(\alpha x)+(-y)+\left(-1-\frac{1}{\alpha}\right) 0, z\right\|^{2} \\
& =\frac{\alpha+1}{\alpha^{2}}\|\alpha x, z\|^{2}+\frac{\alpha+1}{\alpha}\|-y, z\|^{2}-\frac{1}{\alpha}\|\alpha x-(-y), z\|^{2} \\
& =(\alpha+1)\|x, z\|^{2}+\frac{\alpha+1}{\alpha}\|y, z\|^{2}-\frac{1}{\alpha}\|\alpha x+y, z\|^{2}
\end{aligned}
$$

and

$$
\begin{aligned}
\|x-y, z\|^{2} & =\left\|x+\frac{1}{\alpha}(-\alpha y)+\left(-1-\frac{1}{\alpha}\right) 0, z\right\|^{2} \\
& =\frac{\alpha+1}{\alpha}\|x, z\|^{2}+\frac{\alpha+1}{\alpha^{2}}\|-\alpha y, z\|^{2}-\frac{1}{\alpha}\|x-(-\alpha y), z\|^{2} \\
& =\frac{\alpha+1}{\alpha}\|x, z\|^{2}+(\alpha+1)\|y, z\|^{2}-\frac{1}{\alpha}\|x+\alpha y, z\|^{2} .
\end{aligned}
$$

Further, since $\|x, z\|=\|y, z\|$ holds true, the last two equalities imply that

$$
\|\alpha x+y, z\|^{2}=\|x+\alpha y, z\|^{2} \text {, т.e. }\|\alpha x+y, z\|=\|x+\alpha y, z\|,
$$

The latter means that the condition $\mathrm{II}_{3}$ is satisfied.

Lemma 5. Let $L$ be 2-normed space. Then the condition $I I_{10}$ implies the condition $I I_{6}$.

Proof. Let $x_{1}, x_{2}, x_{3}, z \in L$ be such that $\sum_{i=1}^{3} x_{i}=0$ and $\left\|x_{1}, z\right\|=\left\|x_{2}, z\right\|$. For $\alpha=2$, the Lemma 4 implies that 


$$
\left\|2 x_{1}+x_{2}, z\right\|=\left\|x_{1}+2 x_{2}, z\right\|
$$

holds true. Further, since $\sum_{i=1}^{3} x_{i}=0$ we get that $x_{3}=-x_{1}-x_{2}$, thus

$$
\begin{aligned}
\left\|x_{1}-x_{3}, z\right\| & =\| x_{1}-\left(-x_{1}-x_{2}\left(, z\|=\| 2 x_{1}+x_{2}, z\|=\| x_{1}+2 x_{2}, z \|\right.\right. \\
& =\left\|x_{2}-\left(-x_{1}-x_{2}\right), z\right\|=\left\|x_{2}-x_{3}, z\right\|,
\end{aligned}
$$

The latter means that the condition $I_{6}$ is satisfied.

Lemma 6. Let $L$ be 2-normed space. Then the condition $I I_{10}$ implies the condition $I I_{7}$. Proof. Let $x_{1}, x_{2}, x_{3}, x_{4}, z \in L$ be such that $\sum_{i=1}^{4} x_{i}=0$ and $\left\|x_{1}, z\right\|=\left\|x_{2}, z\right\|$ and $\left\|x_{3}, z\right\|=\left\|x_{4}, z\right\|$. Further, since $x_{1}+x_{2}+\left(x_{3}+x_{4}\right)=0$ and $\left\|x_{1}, z\right\|=\left\|x_{2}, z\right\|$ holds true, the Lemma 5 implies that $\left\|x_{1}-x_{3}-x_{4}, z\right\|=\left\|x_{2}-x_{3}-x_{4}, z\right\|$. Further, the condition $I I_{10}$ implies that

$$
\begin{aligned}
& \left\|x_{1}-x_{3}-x_{4}, z\right\|^{2}=\left\|x_{1}-x_{3}-x_{4}+0, z\right\|^{2} \\
& \quad=-\left\|x_{1}, z\right\|^{2}+\left\|x_{3}, z\right\|^{2}+\left\|x_{4}, z\right\|^{2}+\left\|x_{1}-x_{3}, z\right\|^{2}+\left\|x_{1}-x_{4}, z\right\|^{2}-\left\|x_{3}-x_{4}, z\right\|^{2}
\end{aligned}
$$

and

$$
\begin{aligned}
& \left\|x_{2}-x_{3}-x_{4}, z\right\|^{2}=\left\|x_{2}-x_{3}-x_{4}+0, z\right\|^{2} \\
& \quad=-\left\|x_{2}, z\right\|^{2}+\left\|x_{3}, z\right\|^{2}+\left\|x_{4}, z\right\|^{2}+\left\|x_{2}-x_{3}, z\right\|^{2}+\left\|x_{2}-x_{4}, z\right\|^{2}-\left\|x_{3}-x_{4}, z\right\|^{2}
\end{aligned}
$$

and since $\left\|x_{1}-x_{3}-x_{4}, z\right\|=\left\|x_{2}-x_{3}-x_{4}, z\right\|$ and $\left\|x_{1}, z\right\|=\left\|x_{2}, z\right\|$ we get that

$$
\left\|x_{1}-x_{3}, z\right\|^{2}+\left\|x_{1}-x_{4}, z\right\|^{2}=\left\|x_{2}-x_{3}, z\right\|^{2}+\left\|x_{2}-x_{4}, z\right\|^{2} \text {. }
$$

Analogously can be proven the following

$$
\left\|x_{3}-x_{1}, z\right\|^{2}+\left\|x_{3}-x_{2}, z\right\|^{2}=\left\|x_{4}-x_{1}, z\right\|^{2}+\left\|x_{4}-x_{2}, z\right\|^{2} .
$$

Finally, (1) and (2) imply that $\left\|x_{1}-x_{3}, z\right\|=\left\|x_{2}-x_{4}, z\right\|$ and $\left\|x_{2}-x_{3}, z\right\|=\left\|x_{1}-x_{4}, z\right\|$.

The latter means that the condition $\mathrm{II}_{7}$ is satisfied.

Lemma 7. Let $L$ be 2-normed space. Then the condition $I I_{10}$ implies the condition $I I_{8}$.

Proof. Let $x_{1}, x_{2}, x_{3}, z \in L$. Then the condition $I I_{10}$ implies

$$
\begin{aligned}
\| 2 x_{1}+2 x_{2}, z & \left\|^{2}=\right\| x_{1}+\left(x_{2}+x_{3}\right)-\left(x_{3}-x_{2}\right)-\left(-x_{1}\right), z \|^{2} \\
= & -\left\|x_{1}-\left(x_{2}+x_{3}\right), z\right\|^{2}+\left\|x_{1}-\left(x_{3}-x_{2}\right), z\right\|^{2}+\left\|x_{1}-\left(-x_{1}\right), z\right\|^{2} \\
& +\left\|x_{2}+x_{3}-\left(x_{3}-x_{2}\right), z\right\|^{2}+\left\|x_{2}+x_{3}-\left(-x_{1}\right), z\right\|^{2}-\left\|x_{3}-x_{2}-\left(-x_{1}\right), z\right\|^{2} \\
= & \left.-\| x_{1}-x_{2}-x_{3}\right), z\left\|^{2}+\right\| x_{1}-x_{3}+x_{2}, z\left\|^{2}+\right\| 2 x_{1}, z \|^{2} \\
& +\left\|2 x_{2}, z\right\|^{2}+\left\|x_{2}+x_{3}+x_{1}, z\right\|^{2}-\left\|x_{3}-x_{2}+x_{1}, z\right\|^{2},
\end{aligned}
$$

thus 


$$
\begin{aligned}
F\left(x_{1}, x_{2}, x_{3}, z\right)= & \left\|x_{1}+x_{2}+x_{3}, z\right\|^{2}+\left\|x_{1}+x_{2}-x_{3}, z\right\|^{2} \\
& \quad-\left\|x_{1}-x_{2}-x_{3}, z\right\|^{2}-\left\|x_{1}-x_{2}+x_{3}, z\right\|^{2} \\
= & \left\|2 x_{1}+2 x_{2}, z\right\|^{2}-\left\|2 x_{1}, z\right\|^{2}-\left\|2 x_{2}, z\right\|^{2},
\end{aligned}
$$

The later means that the condition $I I_{8}$ is satisfied.

Lemma 8. Let $L$ be 2-normed space. Then the condition $I I_{10}$ implies the condition $I I_{4}$.

Proof. Let $\|x+y, z\|=\|x-y, z\|, x, y, z \in L$ and let $\alpha$ be a real number such that $\alpha \neq 0, \pm 1$. Then the condition $I I_{10}$ implies

$$
\begin{aligned}
\|x-y, z\|^{2} & =\left\|x+\frac{1}{\alpha}(-\alpha y)+\left(-1-\frac{1}{\alpha}\right) 0, z\right\|^{2} \\
& =\frac{\alpha+1}{\alpha}\|x, z\|^{2}+\frac{\alpha+1}{\alpha^{2}}\|-\alpha y, z\|^{2}-\frac{1}{\alpha}\|x-(-\alpha y), z\|^{2} \\
& =\frac{\alpha+1}{\alpha}\|x, z\|^{2}+(\alpha+1)\|y, z\|^{2}-\frac{1}{\alpha}\|x+\alpha y, z\|^{2} .
\end{aligned}
$$

and

$$
\begin{aligned}
\|x+y, z\|^{2} & =\left\|-x-\frac{1}{\alpha}(\alpha y)+\left(1+\frac{1}{\alpha}\right) 0, z\right\|^{2} \\
& =\frac{\alpha+1}{\alpha}\|x, z\|^{2}+\frac{\alpha+1}{\alpha^{2}}\|\alpha y, z\|^{2}-\frac{1}{\alpha}\|x-\alpha y, z\|^{2} \\
& =\frac{\alpha+1}{\alpha}\|x, z\|^{2}+(\alpha+1)\|y, z\|^{2}-\frac{1}{\alpha}\|x-\alpha y, z\|^{2} .
\end{aligned}
$$

Further, since $\|x+y, z\|=\|x-y, z\|$, the last two equalities imply that

$$
\|x+\alpha y, z\|^{2}=\|x-\alpha y, z\|^{2} \text {, i.e. }\|x+\alpha y, z\|=\|x-\alpha y, z\| .
$$

The latter means that the condition $I_{4}$ is satisfied.

Lemma 9. Let $L$ be 2-normed space. Then the condition $I I_{10}$ implies the condition $I I_{2}$. Proof. Let $\|x+y, z\|=\|x-y, z\|, x, y, z \in L$. Then since the proof of Theorem 2 we get

$$
\|x+y, z\|^{2}=2\|x, z\|^{2}+2\|y, z\|^{2}-\|x-y, z\|^{2},
$$

and since $\|x+y, z\|=\|x-y, z\|$, the last equality is equivalent with

$$
\|x+y, z\|^{2}=\|x, z\|^{2}+\|y, z\|^{2} .
$$

The latter means that the condition $I_{2}$ is satisfied.

\section{References}

[1] K. Anevska, R. Malčeski, Characteization of 2-inner product using EulerLagrange type of equality, International Journal of Science and Research (IJSR), ISSN 2319-7064, Vol. 3 Issue 6 (2014), 1220-1222. 
[2] Y. J. Cho, S. S. Kim, Gâteaux derivatives and 2-Inner Product Spaces, Glasnik matematički, Vol. 27(47) (1992), 271-282

[3] C. Diminnie, S. Gähler, A. White, 2-Inner Product Spaces, Demonstratio Mathematica, Vol. VI (1973), 525-536

[4] C. Diminnie, S. Gähler, A. White, 2-Inner Product Spaces II, Demonstratio Mathematica, Vol. X, No 1 (1977), 169-188

[5] C. Diminnie, A. White, 2-Inner Product Spaces and Gâteaux partial derivatives, Comment Math. Univ. Carolinae 16(1) (1975), 115-119

[6] R. Ehret, Linear 2-Normed Spaces, Doctoral Diss., Saint Louis Univ., 1969

[7] S. Gähler, Lineare 2-normierte Räume, Math. Nachr. 28 (1965), 1-42

[8] R. Malčeski, K. Anevska, Characterization of 2-inner product by strictly convex 2norm of modul $c$, International Journal of Mathematical Analysis, Vol. 8, no. 33 (2014), 1647-1652

[9] S. Malčeski, A. Malčeski, K. Anevska, R. Malčeski, Another characterizations of 2-pre-Hilbert space, IJSIMR, e-ISSN 2347-3142, p-ISSN 2346-304X, Vol. 3, Issue 2 (2015), pp. 45-54.

\footnotetext{
${ }^{1)}$ Centre for research and development of education, Skopje, Macedonia E-mail address: samoil.malcheski@gmail.com

${ }^{2)}$ Faculty of informatics, FON University, Skopje, Macedonia

E-mail address: risto.malceski@gmail.com

${ }^{3)}$ Faculty of informatics, FON University, Skopje, Macedonia

E-mail address: anevskak@gmail.com
} 